\title{
Goal-directed access to mental objects in working memory: The role of task-specific feature retrieval
}

\author{
SAbine Schwager and Herbert Hagendorf \\ Humboldt University at Berlin, Berlin, Germany
}

\begin{abstract}
In the present study, we examined the hypothesis of task-specific access to mental objects from verbal working memory. It is currently assumed that a mental object is brought into the focus of attention in working memory by a process of object selection, which provides this object for any upcoming mental operation (Oberauer, 2002). We argue that this view must be extended, since the selection of information for processing is always guided by current intentions and task goals. In our experiments, it was required that two kinds of comparison tasks be executed on digits selected from a set of three digits held in working memory. The tasks differed in regard to the object features the comparison was based on. Access to a new mental object (object switch) took consistently longer on the semantic comparison task than on the recognition task. This difference is not attributable to object selection difficulty and cannot be fully accounted for by task difficulty or differences in rehearsal processes. The results support our assumptions that (1) mental objects are selected for a given specific task and, so, are accessed with their specific task-relevant object features; (2) verbal mental objects outside the focus of attention are usually not maintained at a full feature level but are refreshed phonologically by subvocal rehearsal; and (3) if more than phonological information is required, access to mental objects involves feature retrieval processes in addition to object selection.
\end{abstract}

Everyday cognitive tasks require the simultaneous maintenance and processing of information from the environment, as well as from memory, a function that is usually called working memory. When considering a problem or, for instance, mentally calculating $27 \times 19$, we have to simultaneously generate, maintain, and integrate intermediate results, a task that requires a flexible switching between relevant parts of the memory information held available. At the same time, the parts not currently selected must not be lost but kept available for later access in order to obtain the final result. It is therefore not surprising that working memory abilities appear to strongly determine our intellectual performance (e.g., Engle, Tuholsky, Laughlin, \& Conway, 1999; Kane et al., 2004; Kyllonen \& Christal, 1990; Süß, Oberauer, Wittmann, Wilhelm, \& Schulze, 2002).

An important function required in working memory tasks is the accessing of certain parts of the maintained information. Therefore, in order to achieve the task goal or to realize our intention, we must select relevant information for processing from the pool of available information in working memory, just as we need to select relevant information from the perceived environment. It appears to be this selection requirement that makes working memory tasks especially difficult, whereas a passive memory load has less impact on performance (e.g., Carlson, Sullivan, \& Schneider, 1989; Oberauer, 2001).
Some current models describing working memory performance incorporate a focus of attention that contains the information actually selected for processing, while maintained information that is not selected remains in an activated but less directly accessible state (see Cowan, 1988, 1995, 2001). An alternative approach distinguishes the stores involved in the passive maintenance of memory information from controlled information processing (Baddeley, 1986, 2000; Baddeley \& Hitch, 1974). In Cowan's model, the activated part of long-term memory contains the currently available memory information, while a small selected part of this activated information is in the focus of attention and, so, is the object of current processing. Directing or moving the focus is guided by control processes. According to this idea, accessing elements from working memory can be understood as an attentional-orienting operation within the activated part of long-term memory, similar to the orienting of attention in the perceived environment. Such a view corresponds well to the results of a number of recent studies on visual working memory that have shown parallels between selective comparison tasks executed on displayed versus memorized sets of visual objects. These close relationships are evident in behavioral effects (e.g., Griffin \& Nobre, 2003; Lepsien, Griffin, Devlin, \& Nobre, 2005), as well as in the brain regions involved, under external and internal task conditions (Lepsien \& Nobre, 2006; Nobre et al., 2004).

S. Schwager, sabine.schwager@hu-berlin.de 


\section{Object Selection and Object Switching}

How is flexible access within verbal working memory realized? A useful means for investigating the processes involved in such access is object-switching tasks. Typically, there are two or more elements to be held in memory, while mental operations have to be performed on one of these mental objects. For example, in the first work along this line, Garavan (1998) had his participants simultaneously count two classes of shapes (triangles and rectangles). In this situation, two independent counts had to be kept in working memory. Since the shapes appeared one by one in random order, the count to be updated - that is, the mental object to be worked on - changed randomly from trial to trial. Object-switching costs, measured as the time difference between object repetition (updating the same count twice) and object switch (updating both counts in subsequent steps) trials, are an estimate of the time required to access a new object from the memory set.

Currently, object-switching costs are understood as mirroring the need to select a mental object among several candidates held concurrently in working memory. Oberauer (2002) showed that the amount of object-switching cost depends on the number of mental objects that are potential processing candidates. Furthermore, the number of additional objects - maintained for later recall but marked as irrelevant for the current task - has no influence on object-switching costs. On the basis of these results, Oberauer extended the model proposed by Cowan (1988, 1995, 2001). He distinguished a region of direct access, which contains the potential processing candidates during the current working memory task, from a focus that selects one single object for processing (Oberauer, 2002, 2003, 2005). In the parlance of Cowan's model, both these components would belong more to the focus of attention than to the activated long-term memory. Thus, the region of direct access corresponds to a broad focus of attention, whereas a narrow focus is needed for selective processing. Increasing the number of objects within the region of direct access prolongs object selection, whereas the size of a passive memory set does not affect accessing time (Oberauer, 2001, 2002). In Oberauer's model, mental objects within the region of direct access are considered as memory chunks that can be accessed for controlled processing through object selection. Importantly, in his model, no influences of the task on this selection process and no qualitative differences between mental objects are assumed (except for their being in the focus of attention or not). Most of the existing studies on object switching have relied on this task-independent view: Object selection is the only process necessary to provide a mental object from the memory set for processing. This means that, after an object has been selected, the object is in the focus of attention as a whole and should be available for any mental operation.

In the present article, we argue that the properties of mental objects processed within the focus of attention differ depending on the task that they were accessed for and, as a consequence, that there should be task dependencies of the processes involved in accessing mental objects from working memory. This view is supported by studies on the processing of visual objects in which it has been shown that attention is applied flexibly on the basis of the current task: There appear to be differences in the availability of task-relevant and task-irrelevant information even within the same object (Fanini, Nobre, \& Chelazzi, 2006). We thus prefer a more dynamic view of access to mental objects that allows the integration of task-dependent access processes that provide relevant object information, in addition to the task-independent object selection proposed by Oberauer (2002).

\section{Intentional Object Selection and Task-Dependent Retrieval of Object Features}

The selection of information is guided by current intentions. Thus, when verbal working memory tasks are considered, selection should provide the mental object to be worked on in the processing task. We assume at least two steps or components of such access, one of which is possibly task independent, whereas the other depends on the intended processing task. Access to a specific mental object requires its selection within the given memory set (Oberauer, 2002). As we understand object selection, this process determines which object among the candidates is relevant and makes it directly accessible, over against competing objects, from the region of direct access. Thus, a selected object can, in principle, be subjected to selective mental operations. Under these assumptions, access to mental objects should be independent of the task to be executed afterward.

As was mentioned above, tasks differ with respect to the information that they require to be executed. For example, if the mental object in a given processing step is a number and one has to compare it with a stimulus number presented on the display for identity, only information concerning the number's identity is required. This information can be provided, for example, by the phonological code maintained in working memory through subvocal rehearsal. But this superficial object information would not suffice to judge the numerical relation of the same number to a stimulus number or to execute a mental addition or counting operation. Thus, it probably makes a difference for which task a mental object has to be provided. Whereas purely selecting a certain object might, in fact, be task independent, there should be additional processes that provide task-specific object information.

What are the implications of task-dependent access with respect to the properties of the involved mental objects within and outside the focus of attention? We assume that the object in focus is selected for the execution of a certain mental operation in order to carry out a given working memory task. Therefore, it does not need to be available for any operation other than the one it is accessed for. Consequently, particularly task-relevant object features should be provided when the mental object is accessed, whereas currently irrelevant object features can remain less available. Therefore, the duration of access processes can vary with respect to the kind of features to be provided. We assume further that it is not possible to maintain all kinds of object information at a maximum level of availability without attention. Therefore, mental 
objects removed from the focus are subject to forgetting processes insofar as no attention is paid to them, and this should be the case for at least the time needed to retrieve another object into the focus of attention.

However, since the objects currently not selected are potentially relevant in future processing steps, losing them must be avoided, preferably with the help of a mechanism that does not consume or scarcely consumes attentional resources. The strategy typically used for maintenance of verbal information is articulatory rehearsal. Although such rehearsal has been shown to be initially resource demanding when a new verbal sequence has to be implemented, it appears to proceed relatively automatically afterward (Naveh-Benjamin \& Jonides, 1984). At present, there is some ambiguity concerning the quality of memory representations refreshed by articulatory rehearsal processes. A number of studies have called into question Baddeley's (1986; Baddeley \& Hitch, 1974) original conception of the phonological loop as a phonological buffer accompanied by an articulatory control process. For instance, the effects of irrelevant sound and phonological similarity on short-term memory performance have been shown to be rather more attributable to auditory-perceptual and speech-planning mechanisms than to an independent store that holds phonological representations (e.g., Jones, Macken, \& Nicholls, 2004; Page, Madge, Cumming, \& Norris, 2007). Against the background of these results, we suggest that rehearsal, in the sense of the repeated and automated execution of a speech plan, should result in the maintenance of a kind of motor or premotor verbal representation. For the sake of simplicity, we will use the term phonological when referring to these representations. They are superficial representations of the mental objects to be maintained in working memory and, critically, do not contain semantic features, since these are not necessarily refreshed by articulatory processes.

Given such a set of phonological codes, two things would be necessary to access an object successfully in a given working memory task. First we would have to select the relevant object. Object selection should depend on the properties of the memory set, such as, for example, set size or similarity of the memory elements, but not on the task. If the current task can be carried out on phonological object information, access is successfully concluded at this point. Otherwise - and this is probably the case for most of the typical working memory tasks - a second component of access to additional object information would have to be retrieved from memory. Any semantic or numerical operations would require object features exceeding the information provided by the phonological code. Their retrieval should be time consuming, with the duration depending on the kind of features to be retrieved. We thus hypothesize longer durations of access to a new mental object from the memory set when semantic information is required than when rather superficial or phonological information is sufficient to execute the task at hand.

\section{The Experiments}

Four experiments were conducted to collect evidence for task-dependent retrieval processes involved in accessing mental objects from working memory. Object-switching costs in response times (RTs) served as a measure for the duration of the processes involved in such access.

Differing from most of the previous object-switching studies that used memory updating tasks, such as counting simultaneously (Garavan, 1998; Li, Sun, Wang, Zhang, \& Zhang, 2004; Voigt \& Hagendorf, 2002) or performing mental additions or subtractions on the selected object (Kessler \& Meiran, 2006; Oberauer, 2002), we decided to use object-switching tasks that required only a comparison of selected objects with displayed stimuli. In memory-updating tasks, the mental object not only is accessed, but also is modified during each trial, and objectswitching costs reflect a combination of the time needed for access and for updating. The way we see it, it is not yet clear whether the effects of access and updating on objectswitching costs are simply additive or, rather, determined by complex interactions that can result from the need to overwrite contents just retrieved into the focus of attention. Kessler and Meiran found no statistically significant interactions between object switching and updating, but in fact, the numeric tendencies in their experiments consistently indicated increased costs for an object switch on trials that required updating. Since we concentrated on understanding the processes specific for access to mental objects, we preferred to use an experimental paradigm that required no updating. By doing so, we could also be quite sure that rehearsal proceeded as automatically as possible once a given item set had been memorized.

The experimental task consisted of sequences of comparisons that had to be executed on mental objects selected randomly from a memory set of three digits. In each comparison step, a stimulus number was presented and had to be compared with one of the numbers from the memory set specified by a cue. The memory set did not change over the course of one comparison sequence.

We manipulated the demand on feature retrieval by using comparison tasks that differed only in this respect. The required response was always based on a samedifferent decision and had to be indicated by one of two possible response keys. Furthermore, in order to avoid the confounding effects of object selection difficulty on object-switching costs, equivalent memory sets of equal size were used in each experimental condition.

In the superficial or phonological condition, the comparison sequences consisted of judgments of identity (subjects had to indicate whether the stimulus number matched the mental object in identity). This task differs from classical recognition by requiring the comparison of the probe item with a specific mental object, in contrast to scanning the memory set for the probe at any list position. Thus, this local recognition task probably requires specific object selection, whereas memory scanning does not (see Oberauer, 2005). The semantic condition contained sequences in which numeric object information was necessary to find the correct response.

In Experiment 1, we examined object-switching costs with two tasks that required differing amounts of object information. Experiment 2 was conducted to rule out an explanation of differing object-switching costs in terms 
of the maintenance strategy used by the subjects. Experiment 3 was conducted, on the one hand, to rule out an explanation of the difference based on the use of a (generally faster) memory search, instead of specific access in the local recognition task in Experiment 1, and, on the other hand, to replicate the result with a different kind of object cue. Finally, Experiment 4 was designed to diminish the difference in task difficulty between the comparison tasks, which might be an alternative source of differences in object-switching costs.

\section{EXPERIMENT 1}

Oberauer (2002) explained access to mental objects and the origin of object-switching costs through an object selection process that should provide the selected object for any upcoming mental operation. Experiment 1 was designed to show that a mental object is provided specifically for a currently relevant mental operation by demonstrating task effects on object-switching costs. Since mental operations may differ with respect to the required amount of additional feature retrieval, we would expect differing accessing times, as reflected in object-switching costs, with respect to the task.

In our experiment, we varied feature requirements between tasks while keeping factors influencing object selection constant. In the local recognition (locRec) task, the stimulus number on the display had to be compared directly with the mental object and judged whether it was the same or different. This operation is quite superficial and can be performed immediately on the selected object as it is maintained by rehearsal, probably by comparing the phonological codes of both numbers. In contrast, in the local relation comparison (locRelC) task, one has to compare the relations of both numbers (stimulus and mental object) with the number 4 and indicate whether they are the same (both smaller or both greater than 4) or different (one smaller, one greater than 4). This judgment is based on semantic/numeric information. In this case, the correct selection of the phonological code is not sufficient to make the decision, and additional object features have to be retrieved. However, in both tasks, no selection and retrieval processes are necessary when the object repeats, because all the information needed for the respective task is already in the focus of attention. We therefore would expect higher object-switching costs in locRelC than in locRec sequences.

\section{Method}

Subjects. Twenty-four students at the Institute of Psychology at Humboldt University, Berlin, took part in Experiment 1 (20 female, 4 male). Their ages ranged from 18 to 32 years $(M=22.3, S D=$ 3.66). All had normal or corrected-to-normal vision and received course credit for participation.

Apparatus and Stimuli. Experimental series were conducted using PCs (Pentium 1) with 17-in. monitors and standard PC keyboards. The space bar was used for self-paced starting and continuing. The letter keys "V" and "B" served as response keys for the same/different responses required by the respective comparison task. These had to be pressed with the index fingers of both hands. Presentation of the experimental task and data collection were re- alized using the ERTS package (Beringer, 1999). These technical details were the same for all three experiments and are therefore described only once.

Three geometrical shapes (square, triangle, and circle) served as memory cues. They appeared in black on a light gray background, and each occupied an area of approximately $2.3 \mathrm{~cm}$ in height and width on the computer screen. The numbers to be judged were represented by six digits, the digits $1-6$ for locRec and the digits $1-7$, excluding 4, for locRelC. Digits appeared in white within the respective black shape and were about $1.1 \mathrm{~cm}$ high. At a regular viewing distance of about $60 \mathrm{~cm}$, the stimuli correspondingly occupied visual angles of $2.2^{\circ}$ (shapes) or $1.1^{\circ}$ (digits).

Procedure. The two experimental tasks (locRec and locRelC) were presented in separate blocks. The order of the two experimental parts was balanced over subjects. Written instructions and examples were presented on-screen, and training trials for the respective comparison tasks were completed under the supervision of the experimenter. Both experimental parts were conducted within one session of about $1 \mathrm{~h}$ and were structured as follows.

A short training block (36 trials) familiarized the subjects with the actually relevant comparison task. Pairs of digits were presented centrally on the screen. By pressing one of the response keys, the subjects indicated whether the numbers making up the pair were the same or different or whether they had the same or a different relation to the number 4; that is, in the relation comparison task, subjects indicated same when the two numbers were both smaller or both larger than 4 (e.g., 1 and 3 or 7 and 6 ) and different when one was smaller but the other larger than 4 (e.g., 1 and 5). The stimuli remained on the screen until one of the response keys had been pressed. Feedback for erroneous responses or response misses within the maximum response interval of 3,000 msec was given by a short beep. After $500 \mathrm{msec}$, the next stimulus pair appeared. Digits and responses occurred with equal frequencies and in random order.

The experimental blocks began with an instruction phase including two training examples. Then sequences of comparisons using individual elements from working memory had to be completed. Figure 1 demonstrates the course of a locRelC sequence. Sequences in the locRec blocks were identical, with the exception that the digits involved ranged from 1 to 6 . The three digits in the actual memory set were presented in a vertical arrangement simultaneously with the three shapes. Each shape served as cue for an individual number (mental object) from the memory set. After memorizing the three combinations of shape and number, the subjects started the sequence of comparisons by pressing the space bar. Following a blank screen $(500 \mathrm{msec})$, a white digit within a black shape appeared centrally. The actual task (locRec or locRelC) had to be performed on the presented number and the mental object specified by the shape. When one of the response keys was pressed, the stimulus disappeared, followed by the next stimulus, after a response-stimulus interval of $500 \mathrm{msec}$. A sequence consisted of seven comparisons, with random switching between the three mental objects (resulting in about $66 \%$ object switch trials). Error feedback was given as in the training block. In the locRelC condition, the numbers of the memory set had to be typed at the end of each sequence in order to ensure that the subjects had maintained the numbers over the sequence correctly. The typed numbers appeared within the shapes presented vertically, as at the beginning of the sequence. For false entries, an " $X$ " appeared instead. After $1,000 \mathrm{msec}$, the next sequence began with the presentation of a new memory set. In each of the two parts of the experiment, the subjects completed three experimental blocks of 25 sequences each. Given an equal frequency of possible transitions between the three mental objects, there were 300 trials following an object switch and 150 trials on which the same object was used repeatedly, for each of the two comparison tasks.

The six possibilities for ordering the three shapes (i.e., of combining shapes and positions within the memory set) were balanced across subjects, and each subject saw a constant ordering of shapes over the whole experiment. Memory sets were constructed and ordered as follows. For each memory set, three numbers were chosen 


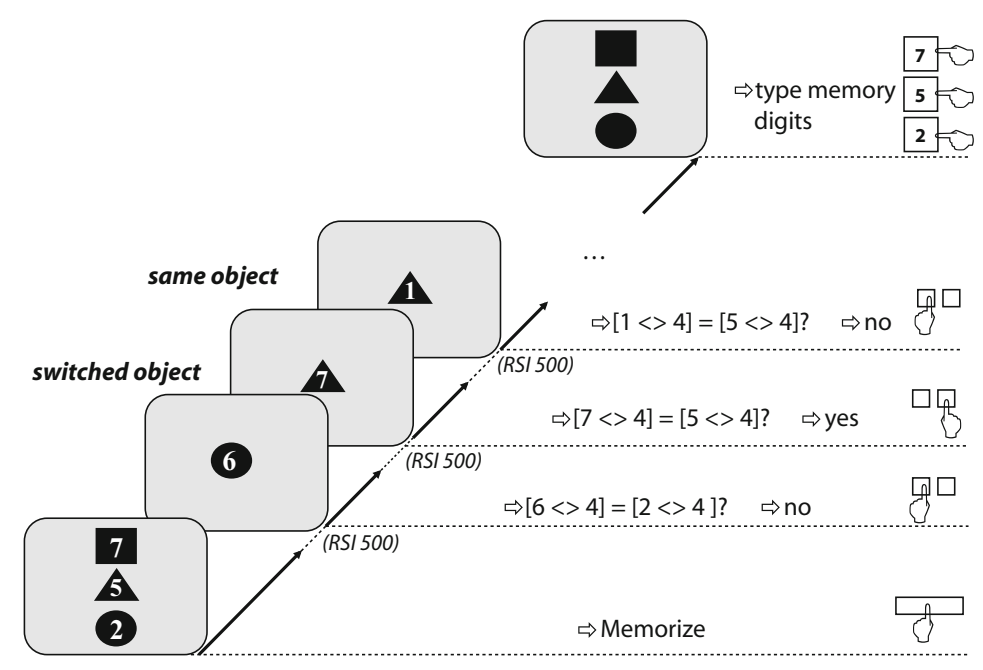

Figure 1. Example of a local relation comparison sequence in Experiment 1 (each sequence consisted of seven comparisons). RSI, response-stimulus interval.

randomly either between 1 and 6 (locRec) or between 1 and 7, except 4 (locRelC). There were no repetitions of numbers within a memory set and no repetitions of memory sets within an experimental part. The stimuli presented for comparison in the sequences were ordered quasirandomly, with the following restrictions: equal frequencies of possible transitions between the three mental objects, as well as of the transitions between the six different numbers involved; no immediate repetitions of the digit presented; and equal frequencies of same and different responses. Moreover, the proportion of numbers presented as stimuli that were simultaneously contained in the current memory set was adjusted for the locRelC task: For the locRec task, all stimuli requiring same responses were contained in the memory set; stimuli for comparisons that required different responses were also contained in the memory set, with a proportion of 2:3 (since these were chosen randomly from the five numbers not matching the mental object actually selected). In sum, the proportion of stimulus numbers contained in the current memory set to numbers not contained in the current memory set was 7:3 in the locRec condition. This relation was reproduced when constructing the sequences in the locRelC condition.

\section{Results}

The critical measure in all the experiments was objectswitching costs reflected in the difference in RT between comparisons following an object switch and those executed on the same mental object as in the step before (object repetition). Statistical analyses (mainly withinsubjects ANOVAs) were generally based on median RTs computed per subject and condition. Effects with an alpha error probability of less than 5\% were considered statistically significant. Comparison steps with incorrect responses or following an incorrect response were excluded from the analyses of RTs. Error rates were analyzed in the same way as RTs (with trials without a response treated as errors). Error rates in our paradigm reflect a combination of failures to access the relevant mental object and errors in the respective comparison operation itself. Therefore, experimental hypotheses were tested mainly on RT effects. Error rates were considered in order to rule out confounding influences of possible speed-accuracy tradeoffs. The figures demonstrating the experimental results contain within-subjects confidence intervals for the critical conditions (Loftus \& Masson, 1994).

Exclusion of data points. The data of 1 participant had to be excluded because she performed at chance level (50\% errors) in the locRelC blocks. We applied an additional criterion on the locRelC blocks: In reproducing the numbers of the memory set, the subjects had to be completely correct in at least $50 \%$ of the sequences and to reproduce two out of the three numbers correctly in at least $75 \%$ of the sequences. All of the remaining 23 subjects met this recall criterion. Statistical analyses were run on all sequences in the locRec condition and on sequences in the locRelC condition that were finished with correct recall of at least two numbers of the memory set. The same recall criteria were used in Experiments 2, 3, and 4.

In the locRec condition, the restrictions for trial construction led to the absence of repeated same responses on object repetition trials (because the stimulus number was never repeated). To avoid misleading effects of the proportion of response repetitions on the estimation of objectswitching costs in the locRec condition, we excluded trials with a repeated same response in combination with an object switch as well.

Response times. A within-subjects ANOVA including the experimental factors of task (locRec vs. locRelC) and object switch (object repetition vs. object switch) revealed main effects of both factors [task, $F(22,1)=88.67, p<$ $.001, \eta^{2}=.80$; object switch, $F(22,1)=57.09, p<.001$, $\left.\eta^{2}=.72\right]$ and an interaction between them $[F(22,1)=$ 46.94, $p<.001, \eta^{2}=.68$ ]. Comparing relations took, on average, longer than comparing identities; switching between mental objects led consistently to a cost in RT, and this time cost was larger when relation comparisons were required (see Figure 2). 


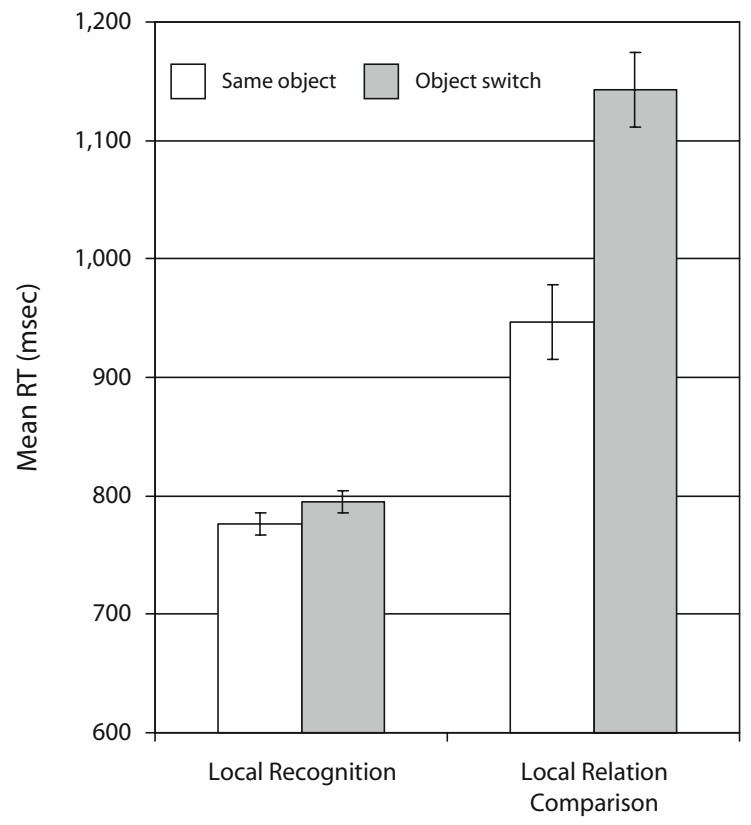

Task

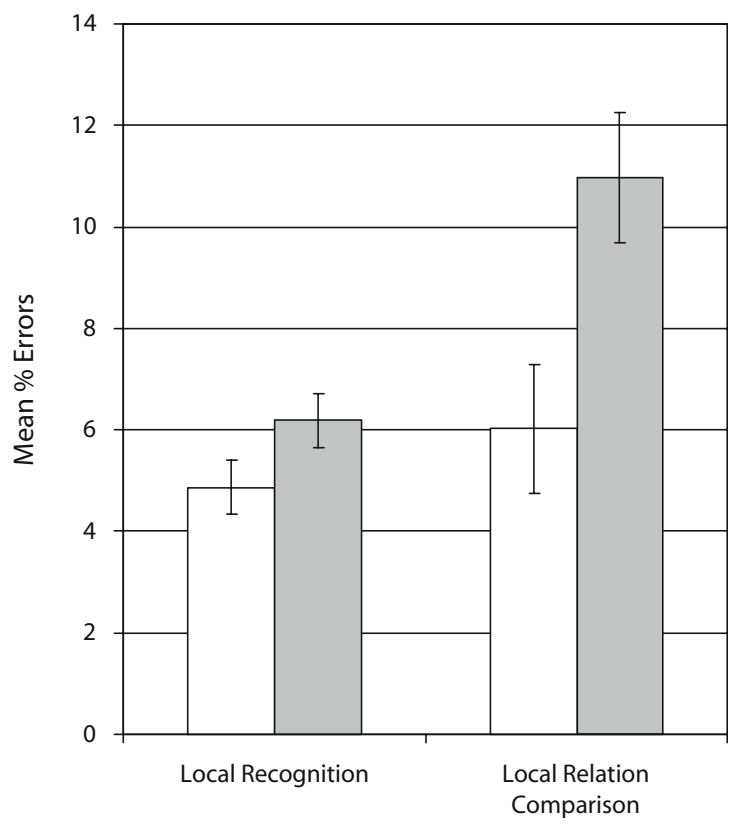

Task

Figure 2. Mean response times (RTs) and error rates for the two comparison tasks (local recognition and local relation comparison) in Experiment 1 in same-object and object switch trials. Error bars mark the confidence intervals for the within-subjects effects of object switch.

The two comparison tasks differed, as was intended, with respect to the information required of the mental object, and the additional time needed to retrieve semantic/ relational information increased object-switching costs in the locRelC task. However, although the final decision (same or different) was kept identical, the displayed stimuli had also to be processed differently. In each trial of the locRelC blocks, the relation of the stimulus digit to the number 4 had to be determined, which is more difficult than just using the digit as a recognition probe. Moreover, comparing two relations might itself be more difficult than comparing identities. An estimate of the effect of task difficulty is provided by the performance difference between the two tasks on same-object trials $[F(22,1)=41.52, p<$ $\left..001, \eta^{2}=.65\right]$. To adjust the difference in switching costs for task difficulty, we reanalyzed switching costs after normalizing the raw RTs by subtracting the individual mean RT and dividing by the individual standard deviation observed on same-object trials per task. ${ }^{1}$ Consistent with our hypothesis, object-switching costs remained higher for the locRelC task $[t(22)=6.25, p<.001]$.

Errors. On average, $7.47 \%$ of the trials were responded to incorrectly $(S D=3.12)$. Error rates were statistically analyzed analogously to the RTs. The ANOVA revealed significant main effects of task $[F(22,1)=12.37, p=$ $\left..005, \eta^{2}=.36\right]$ and object switch $[F(22,1)=33.29, p<$ $\left..001, \eta^{2}=.60\right]$ and a significant interaction between task and object switch $\left[F(22,1)=9.55, p=.046, \eta^{2}=.30\right]$, indicating a higher error cost for object switch trials in the locRelC condition (see Figure 2).
The statistical effects found in the error rates correspond to the RT results. Thus, it is not likely that the interaction of task and object switch was the result of a speed-accuracy trade-off.

\section{Discussion}

Experiment 1 revealed larger time costs for a switch between mental objects when a comparison of numerical relations had to be performed than when the numbers had to be simply compared for identity. This difference appears not to be directly attributable to the higher difficulty of the locRelC task. It remained statistically significant after normalizing the raw RTs for task difficulty. This is consistent with the finding that the difficulty of mental operations does not affect object-switching costs when retrieval demand is kept constant (Voigt \& Hagendorf, 2002).

The data in the first experiment therefore support the hypothesis that task-dependent feature retrieval processes are involved in accessing mental objects from working memory. The concentric working memory model proposed by Oberauer (2002) would not be sufficient to account for the results of Experiment 1: Since the conditions of object selection (set size, properties of the memory elements) were kept constant, there should be no differences in object selection difficulty and, thus, in object-switching costs. Since mental tasks differ with respect to the features they require, the time demand for the additional retrieval processes varies independently of the difficulty of object selection. 
Furthermore, the results can be interpreted as evidence supporting the assumption that mental objects within and outside the focus of attention differ in more respects than the amount of attention that is applied to them: Objects outside the focus appear to be available only with a reduced feature set. This is indicated by the difference in objectswitching costs between the (superficial/phonological) locRec task and the (semantic/numeric) locRelC task. If the numeric features of the mental objects required by the locRelC task had been kept active within the region of direct access, object-switching costs should have been independent of the task. Moreover, the subjects in our experiment knew in advance which kind of object features would be relevant in the upcoming 75 comparison sequences. In this situation, they should have tried to maintain semantic objects if they were able to. ${ }^{2}$ In fact, our result corresponds well to the assumption that rehearsal allows maintenance mainly of phonological information, whereas the additional object features are subject to forgetting processes as soon as attention is drawn away.

One limitation in interpreting the difference in objectswitching costs in Experiment 1 arises from a strategy reported by about half of the subjects. They felt that the locRelC sequences were easier to accomplish by maintaining the three numbers of the actual memory set for later recall but rehearsing their three relations to the number 4 for access during the comparison sequence. Consequently, the higher object-switching costs in the locRelC condition may, in fact, partly go back to more difficult object selection, because these subjects kept three numbers plus three relations (in sum, six mental objects instead of three) in working memory. There are some arguments against this interpretation. First, the effects found in Experiment 1 were consistent across the subjects, regardless of the strategy used. There was also no other difference between the data from the subjects who had used the alternative strategy and from those who reported having worked according to the original instructions. Second, current theories of working memory would not necessarily predict a set size effect in this situation, because the items maintained for later recall would constitute a rather passive memory set and, therefore, would not belong to the region of direct access. Set size effects on RTs and object-switching costs are usually absent when the respective memory elements are not potential objects of the current processing task (Oberauer, 2001, 2002).

\section{EXPERIMENT 2}

The second experiment was conducted to clarify the influence of the strategy reported by some subjects on the amount of object-switching costs in the locRelC sequences. In Experiment 2, the locRelC condition in Experiment 1 was repeated, with all the subjects maintaining only three relations. This strategy was encouraged by starting each sequence with the subjects' judging the relations of the three numbers of the memory set and being explicitly instructed to exclusively maintain these relations. At the end of each sequence, the relations had to be recalled, instead of the numbers. If the advantage in object access achieved by strategy users in the locRelC condition in Experiment 1 suffered from additional memory load through the numbers of the memory set, we should, on average, find lower object-switching costs in Experiment 2, because here the subjects merely maintained relations. If, alternatively, the difference in object-switching costs between the locRec and locRelC conditions in Experiment 1 was determined by the need to retrieve semantic object information on the basis of a phonological code, we would expect no difference between the locRelC blocks conducted in Experiments 1 and 2 .

\section{Method}

Subjects. Twenty-one students at the Institute of Psychology at Humboldt University, Berlin, took part in Experiment 2 (19 female, 2 male). Their ages ranged from 20 to 34 years $(M=23.9, S D=$ 3.83). All had normal or corrected-to-normal vision and received course credit for participation.

Procedure. The procedure in Experiment 2 was identical to that in the locRelC part of Experiment 1, except for some changes at the beginning and end of the sequences (see below). Instructions were presented on-screen, and training trials were performed under the supervision of the experimenter. The procedure differed from that in the locRelC condition in Experiment 1 in the following respects. When the numbers of the memory set were presented (three numbers, each centered within a shape; see Figure 1), the subjects first had to indicate their relation to the number 4 by pressing " $G$ " (larger) or "K" (smaller) sequentially for each of the three numbers. After the response key had been pressed, the correct relation was presented in written form ("größer" or "kleiner") on the right-hand side of the respective number-shape combination. If the wrong response key had been pressed, an exclamation mark accompanied the correct relation presented beside the number. The subjects started the comparison sequences themselves by pressing the space bar so that they could take enough time to memorize the correct relations in advance. After a sequence of seven comparisons, the three shapes reappeared on the screen, as in Experiment 1. Instead of the numbers, the subjects had to type in the three relations, again using the "G" and " $\mathrm{K}$ " keys. Following correct responses, the relation ("größer"/"kleiner") appeared beside the respective shape; otherwise, an " $\mathrm{X}$ " indicated an error. The subjects completed 10 training sequences and two experimental blocks of 25 sequences each (providing 200 trials with object switch and 100 trials with object repetition, in total).

\section{Results}

Exclusion of data points. None of the 21 data sets had to be excluded from analysis due to the recall criterion's not being met (see Experiment 1) or for containing too many errors.

Response times. Experiment 2 yielded significant object-switching costs $\left[F(20,1)=136.97, p<.001, \eta^{2}=\right.$ .87]. At an average of $354 \mathrm{msec}$, they were even larger than the costs found in the locRelC condition in Experiment 1 (197 msec). Accordingly, a common ANOVA on the data for the relation comparison sequences in both experiments, with object switch as a within-subjects factor and experiment as a between-subjects factor, yielded a significant interaction between both factors $[F(42,1)=$ $\left.15.67, p<.001, \eta^{2}=.27\right]$. As Figure 3 shows, the interaction arose from a combination of shorter RTs on sameobject trials and longer RTs on object switch trials in Experiment 2. However, these differences did not approach statistical significance when tested individually [object 


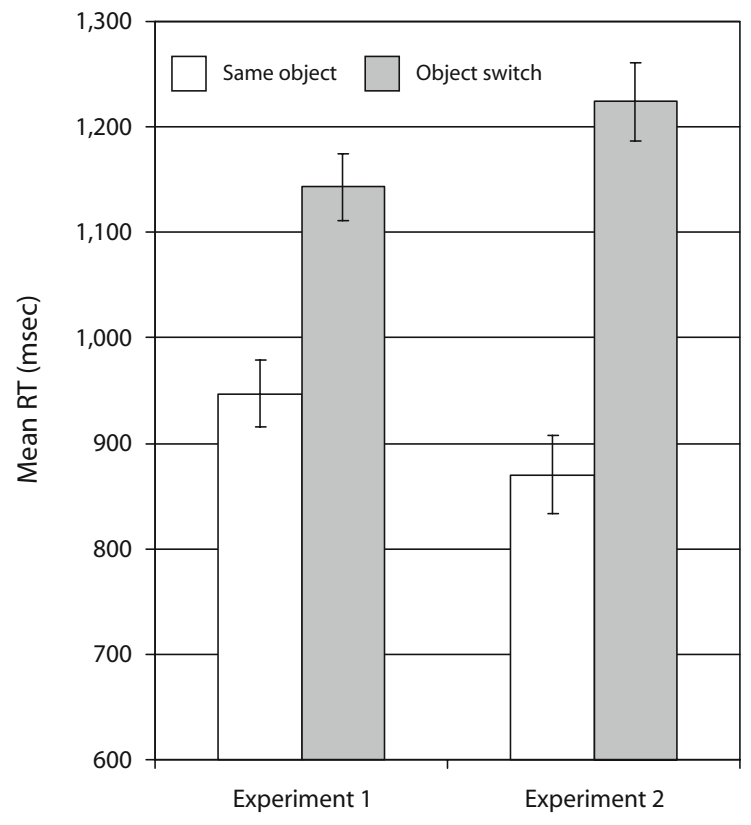

(Local Relation Comparison)

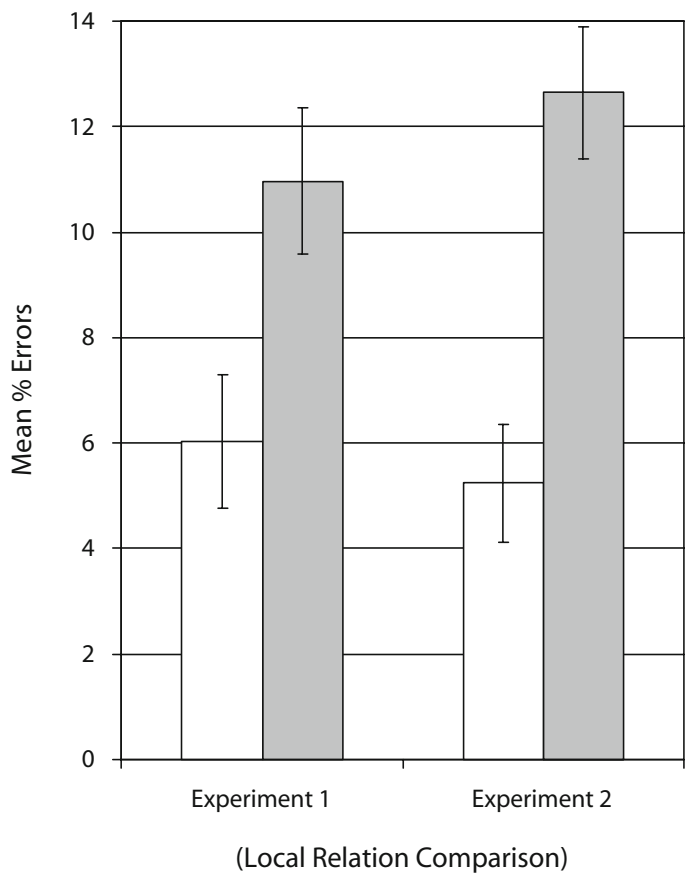

Figure 3. Mean response times (RTs) and error rates in the local relation comparison sequences in Experiments 1 and 2 on sameobject and object switch trials.

repetition, $F(42,1)=2.32, p=.135, \eta^{2}=.05 ;$ object switch, $\left.F(42,1)=1.45, p=.235, \eta^{2}=.03\right]$.

Errors. The mean error rate was $10.21 \%(S D=4.72)$. The effects found in the statistical analyses correspond to the RT data and do not show an influence of a strategic speed-accuracy trade-off. More errors were made following an object switch than following an object repetition $\left[F(20,1)=64.27, p<.001, \eta^{2}=.76\right]$. The interaction between object switch and experiment was not significant in the error rates $\left[F(42,1)=3.12, p=.096, \eta^{2}=.07\right]$ but showed a similar tendency (higher error costs for an object switch in Experiment 2).

\section{Discussion}

Significant object-switching costs were found in Experiment 2. They were larger than the costs obtained in the locRelC condition in Experiment 1. We are therefore able to state that the object-switching costs in the locRelC condition in Experiment 1 did not result from an increased memory load when the subjects additionally rehearsed the three relations needed from the memory set. Otherwise, since only the relations, not the numbers, had to be maintained in Experiment 2, object-switching costs should have been smaller here than in Experiment 1.

But what causes the increased object-switching costs in the locRelC task? We will discuss two alternative interpretations of the results of the first two experiments that do not assume access to semantic information as a source of increased object-switching costs. First, since the locRelC task itself is simply more difficult, object-switching costs in its context might be increased regardless of feature de- mands on the selected object or maintenance strategies. More difficult means, here, that the displayed digit has to be processed to determine its relation to the number 4 and that relations have to be compared, instead of simple identities. However, the data from Experiment 1 showed that eliminating this task-specific time and effort (mirrored in the RTs on same-object trials) did not eliminate the difference in object-switching costs between the locRec and locRelC tasks.

Second, the strategy reported in Experiment 1 and given as an instruction in Experiment 2 suggests that the subjects compared relations by matching a phonological representation from memory (i.e., a higher or a lower code) to a phonological representation of the relation of the displayed digit to the number 4 . In this situation, one would expect object-switching costs similar to the costs found in the locRec task. Instead, they were as high as the costs found for subjects who accessed the relation of the selected mental object to the number 4 on every single trial, as they were instructed to do in Experiment 1. Given a phonological matching strategy, the procedure in Experiment 2 differed from that in the locRec condition in Experiment 1 in just one respect: the need to determine the relation of the displayed digit to the number 4 and to convert this information into a phonological code. However, these operations affected same-object trials as well as object switch trials, since the displayed digits were never repeated on successive trials. This additional effort should have been neutralized by the normalization procedure used in Experiment 1. At this point, we cannot finally rule out the possibility that effects of task difficulty on 
access might manifest in situations in which accessing the information from the displayed stimulus and from the mental object have to proceed simultaneously, as is possibly the case on object switch trials. We addressed this issue in Experiments 3 and 4.

If it is supposed that task difficulty is not the main source of the difference in object-switching costs between the two tasks, there remain two interpretations of the results of Experiment 2. If the subjects used a phonological comparison strategy, they had to convert the relation of the displayed digit into a phonological code on object switch trials. On same-object trials, in contrast, the phonological code of the mental object remaining in the focus of attention might already have activated its meaning, and the comparison could be based on semantic information, which was available before the phonological code of the displayed digit. However, if the need to convert a relation into a phonological code has such a large effect on RT, subjects who followed the instructions in Experiment 1 were quite unlikely to have used a phonological strategy in the locRelC task. Otherwise, they should have shown much larger object-switching costs resulting from the additional conversion of the relation determined from the mental object.

The other interpretation is that, as initially intended, locRelC is based on semantic information (i.e., on the meaning of the relations, but not their phonological codes). Since articulatory rehearsal does not refresh semantics outside the focus of attention, the retrieval of semantic information increases the time required to access a new mental object, regardless of the "proximity" of the respective phonological code to the semantic information needed for processing. The data available from the first two experiments do not allow a decision to be reached between these two possible mechanisms. In principle, the different strategies (phonological vs. semantic comparison) may indeed result in differing access processes. However, both explanations share the characteristic that the advantage on same-object trials results from the direct availability of semantic information for the object in the focus of attention.

\section{EXPERIMENT 3}

Two additional factors may have led to especially low costs when mental objects were accessed in the locRec task in Experiment 1 and, as a consequence, to an overestimation of the difference in switching costs between the two tasks. First, on same trials, the mental object itself was displayed on the screen, which should have facilitated access, especially when a new object had to be selected. There was no comparable facilitation in the locRelC task, because the mental object to be selected was never presented as a stimulus. ${ }^{3}$ Second, on different trials, some of the stimulus numbers were not members of the actual memory set and could, in principle, have been responded to without accessing the specified object (because nonset stimuli were different from all the members of the memory set). These objections were supported when we reanalyzed the locRec data from Experiment $1 .{ }^{4} \mathrm{After}$ post hoc exclusion of same trials and trials with nonset stimuli, objectswitching costs were still larger in the locRelC task, but the interaction no longer approached statistical significance $\left[F(22,1)=3.61, p<.071, \eta^{2}=.14\right]$.

Against this background, Experiment 3 should clarify whether the higher object-switching costs in the locRelC task can be found when all the stimulus digits in the locRec task are elements of the memory set currently maintained (i.e., when every different decision must be based on access to the specified mental object). Furthermore, same trials of the locRec condition are a priori excluded from analysis.

Second, in Experiment 3, spatial positions, instead of shapes, specified the mental object to be accessed. Replicating the critical result with a different kind of object cue is important in order to rule out the possibility that it was just a consequence of the specific task layout in Experiment 1.

Spatial position has served as an object cue in many object-switching studies, and there are data that show that switching between mental objects on the basis of spatial position cues costs less than switching on the basis of other kinds of arbitrary cues, such as color (Oberauer, 2003). In the absence of a proven explanation, one might speculate about the reasons for this easier access via spatial position. For example, associations between spatial positions and serial positions might be easier to maintain and to use for object selection than associations between colors or shapes and serial positions. Essentially, if access via spatial position cues was generally easier in our study too, finding a comparable increase in object-switching costs in the locRelC task could be one more indication of the independence of the affected processes.

\section{Method}

Subjects. Twenty-four students at the Institute of Psychology at Humboldt University, Berlin, took part in Experiment 2 (19 female, 5 male). Their ages ranged from 18 to 40 years $(M=24.3, S D=$ 5.63). All had normal or corrected-to-normal vision and chose between course credit or payment $(€ 7)$ prior to participation.

Stimuli. The presentation differed from that in Experiment 1 in the following respects. No shapes were presented. The screen position of the stimulus digit indicated which number to select from the memory set as the mental object for the actual comparison. The vertical arrangement of the three possible screen positions occupied a visual angle of about $14.2^{\circ}$, given a regular viewing distance of about $60 \mathrm{~cm}$. Digits were presented in light gray color within a white square (side length, $6 \mathrm{~cm}$ ) placed centrally on a dark gray screen (see Figure 4).

Procedure. The experiment consisted of the locRec and locRelC parts, which followed a procedure identical to that in Experiment 1, except that mental objects were cued by their spatial position (see Figure 4) and the pool of stimulus digits in the locRec condition was restricted to elements of the actual memory set, to prevent any use of a global recognition strategy. The order of the locRec and locRelC conditions was balanced across subjects.

\section{Results}

Exclusion of data points. The data of 1 subject had to be excluded because she did not meet the recall criterion in the locRelC blocks. 

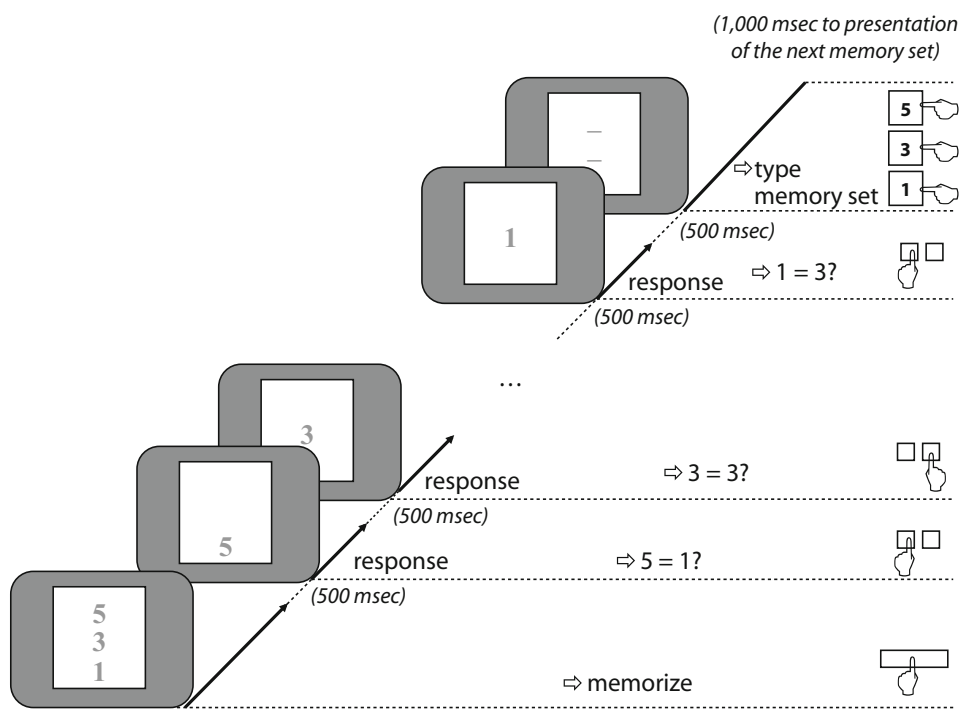

Figure 4. Example of a local recognition sequence in Experiment 3 (each sequence consisted of seven comparisons).

Response times. A first ANOVA with task (locRec vs. locRelC) and object switch (object repetition vs. object switch) as factors revealed main effects of both factors [task, $F(22,1)=6.53, p=.018, \eta^{2}=.23$; object switch, $\left.F(22,1)=32.66, p<.001, \eta^{2}=.60\right]$ and an interaction between them $\left[F(22,1)=17.96, p<.001, \eta^{2}=.45\right]$. Comparing relations took, on average, longer than judging identities; switching between mental objects consistently led to a cost in RT; and this time cost was larger when local relation comparisons were required (129 vs. $37 \mathrm{msec}$; see Figure 5). There was no effect of task difficulty on sameobject trials in Experiment $3(F=1)$. Thus, a comparison of the object-switching costs computed from normalized RTs did not alter the result $[t(22)=3.70, p<.001]$.

We then subjected the RT data in Experiment 1 (without nonset and same trials in the locRec task) and Experiment 3 to a common ANOVA, adding type of cue as a betweensubjects factor. This analysis yielded faster responses [type of cue, $\left.F(44,1)=37.69, p<.001, \eta^{2}=.46\right]$ and lower object-switching costs [type of cue $\times$ object switch interaction, $F(44,1)=16.18, p<.001, \eta^{2}=.27$ ] when mental objects were specified by spatial position. Importantly, the three-way interaction between type of cue, task, and object switch was not significant $\left[F(44,1)=1.95, p=.169, \eta^{2}=\right.$ $.04]$, showing that the effect of task on object-switching costs was not modulated significantly by the type of cue.

Errors. On average, $6.04 \%$ of the trials were responded to incorrectly $(S D=3.61)$. The ANOVA revealed a significant main effect of task $[F(22,1)=29.05, p<.001$, $\left.\eta^{2}=.57\right]$; the effect of object switch and the interaction were not significant (Figure 5).

\section{Discussion}

In Experiment 3, the main result of Experiment 1 was replicated: Object-switching costs were larger when the mental object had to be accessed for the locRelC task, as compared with the locRec task. We controlled a priori for factors that would artificially reduce object-switching costs in the locRec task so that this difference would be reliable. The data also provide arguments against an explanation of the difference in object-switching costs based on task difficulty. First, the fact that there was statistically no effect of task on same-object trials points to a rather negligible influence of task difficulty in this experiment. Second, the remarkably shorter RTs and lower objectswitching costs with spatial position as the object cue show that Experiment 3 was generally less effortful than Experiment 1 with shape as the object cue. However, the effect of task on object-switching costs was not reduced significantly by the type of cue. In fact, the difference in switching costs was even numerically larger under the easier conditions in Experiment 3 and should, therefore, not have been caused by general difficulty.

\section{EXPERIMENT 4}

In Experiment 3, no effect of task difficulty on RTs on same-object trials was observed, and increased objectswitching costs in the locRelC task were found under conditions generally easier than those in Experiment 1. However, these are only indirect arguments for a negligible influence of task difficulty on the retrieval component of access to mental objects. In Experiment 4, we intended to equalize the demands on stimulus processing in the two tasks used. If the difficulty of extracting the relevant information from the displayed stimulus increases object-switching costs in the semantic comparison task, we would expect no difference in object-switching costs when this difficulty is comparable. If it is the need for additional feature retrieval when a new mental object is 


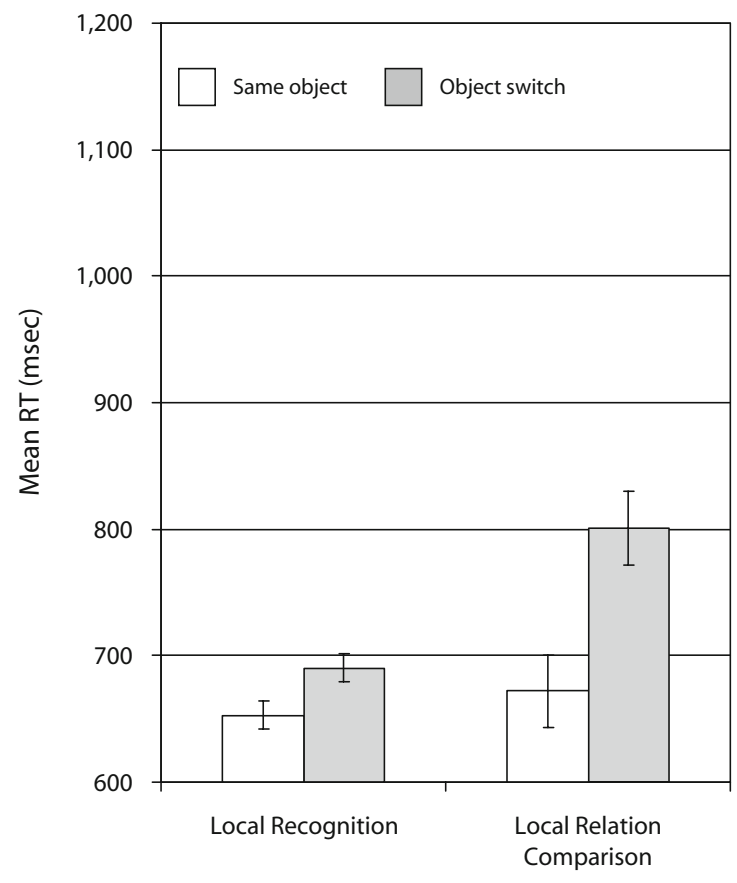

Task

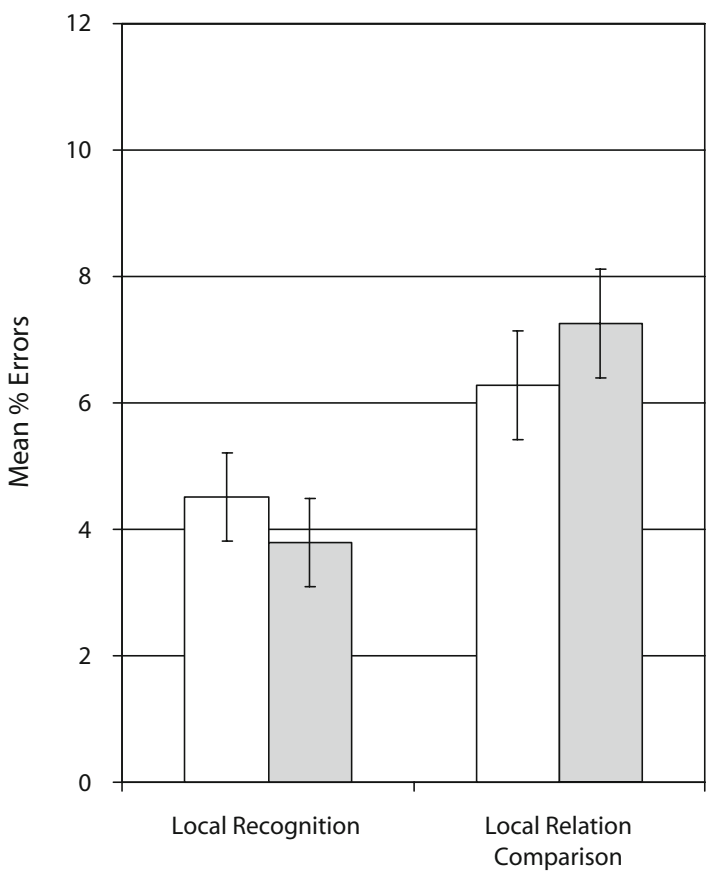

Task

Figure 5. Mean response times (RTs) and error rates for the two comparison tasks (local recognition and local relation comparison) in Experiment 3 on same-object and object switch trials.

accessed, we would again expect higher object-switching costs when semantic information is required.

In a pilot study (with a memory set size of one), we contrasted the original locRec task with a locRec task in which the identification of the displayed number was hampered to some extent and with two semantic tasks: the locRelC task and a simple numeric magnitude comparison (locNumC) task. A data analysis revealed comparable RTs in the difficult locRec task and the locNumC task. We therefore included these tasks in Experiment 4. Using the simple numeric comparison task provided the possibility of replicating the critical result with a different semantic task. Second, comparing numeric values does not require the determination of specific relations first and is, therefore, more similar to the comparison operation in the locRec task. Third, a locNumC task does not allow strategies that rely on phonological codes and, so, provided the possibility of ruling out the alternative interpretations of Experiments 1 and 3.

\section{Method}

Subjects. Twenty students at the Institute of Psychology at Humboldt University, Berlin, took part in Experiment 4 (15 female, 5 male). Their ages ranged from 19 to 34 years $(M=22.6, S D=$ 3.56). All had normal or corrected-to-normal vision and received course credit for participation.

Stimuli. The presentation in Experiment 4 paralleled the presentation in Experiment 3 in its main points. Spatial position served as the object cue. Digits were presented in light gray color within a central white square (side length, $6 \mathrm{~cm}$ ) marked by a frame. Stimulus digits in the locRec task were rotated by randomly varying angles of $60^{\circ}, 120^{\circ}, 180^{\circ}, 240^{\circ}$, and $300^{\circ}$. Furthermore, they were smaller $(0.6 \mathrm{~cm}$ in size) and of a lighter gray color, as compared with the digits presented for memorizing and with the stimulus digits in the locNumC task. In the locNumC task, the subjects had to indicate whether the presented number was smaller or larger than the specified mental object. The "C" (smaller) and "N" (larger) keys were marked correspondingly. This mapping should be compatible with the spatial effects of the mental number line and was applied to all the subjects to avoid additional interference effects.

Procedure. The experimental procedure was analogous to the one in Experiment 3. The order of the locRec and locNumC conditions was balanced across subjects.

Memory sets in the locNumC condition contained digits between 2 and 8 , with a minimum difference of two between all members of a memory set. Memory sets did not repeat within at least five successive sequences. The stimuli presented for comparison were drawn quasirandomly, with the following restrictions: equal frequencies of possible transitions between the three mental objects, no immediate repetitions of the digit presented, and equal frequencies of smaller and larger responses. Furthermore, they were never members of the memory set but, where possible, were drawn from the intermediate numbers. Thus, except for the stimulus digits that had to be smaller than the smallest or larger than the largest set member, the stimuli were always ambiguous, in that the correct response and comparison had to be based on the specified object.

\section{Results}

Response times. ${ }^{5}$ RTs on same-object trials did not differ between the two tasks $(F=1)$, indicating an approximately equal difficulty. An ANOVA with task (locRec vs. locNumC) and object switch (object repeti- 
tion vs. object switch) as factors revealed main effects of both factors [task, $F(19,1)=12.42, p=.002, \eta^{2}=.40$; object switch, $\left.F(19,1)=51.50, p<.001, \eta^{2}=.73\right]$ and an interaction between them $[F(19,1)=28.49, p<.001$, $\left.\eta^{2}=.60\right]$. Comparing numeric values took, on average, longer than judging identities. Switching between mental objects consistently led to a cost in RT, and this time cost was larger when numerical comparisons were required (193 vs. 65 msec; see Figure 6).

Errors. On average, $6.04 \%$ of the trials were responded to incorrectly $(S D=3.61)$. The ANOVA revealed a significant main effect of task $[F(19,1)=5.63, p<.028$, $\left.\eta^{2}=.23\right]$ and an interaction of object switch and task $\left[F(19,1)=15.15, p=.001, \eta^{2}=.44\right]$. The main effect of object switch was not significant $[F(19,1)=3.02, p=$ $\left..099, \eta^{2}=.14\right]$ (see Figure 6).

\section{Discussion}

In Experiment 4, larger object-switching costs were obtained with the locNumC task, as compared with the locRec task. The difficulty of extracting the relevant information from the displayed digit was kept approximately equal in the two tasks. If this difficulty had been responsible for the difference in object-switching costs in the preceding experiments, approximately equal object-switching costs should have been obtained in Experiment 4. We found, instead, that the two tasks differed only in the situation of an object switch - that is, in the processes necessary when a new object had to be accessed from working memory. The result is consistent with our hypothesis of

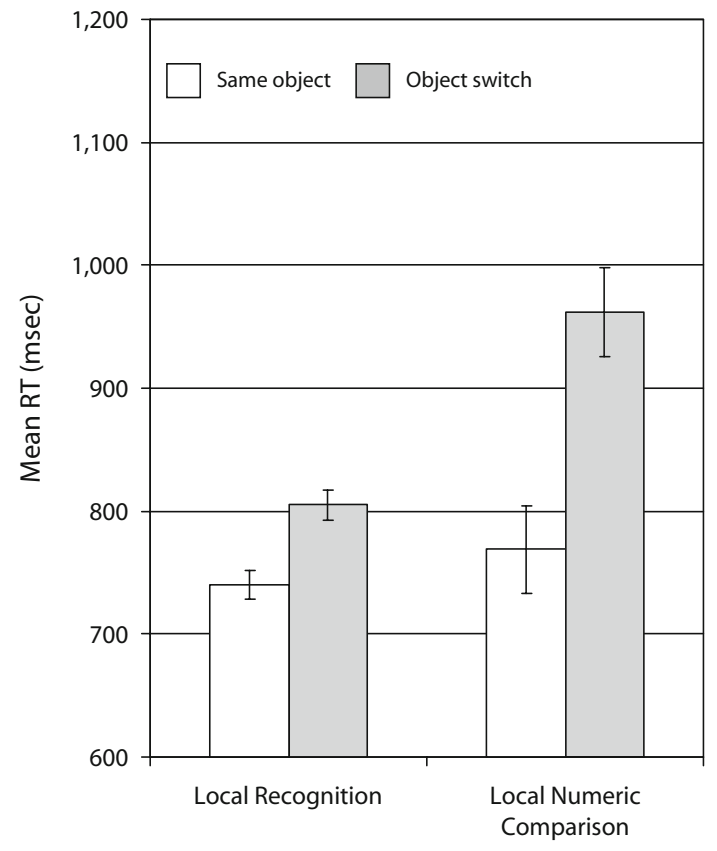

Task task-dependent feature retrieval that proposes additional retrieval processes if the task requires more information than maintenance rehearsal outside the focus of attention can provide. In our case, numeric comparison requires semantic information that is not necessary for local recognition and, thus, leads to longer accessing times when the mental object is switched.

Since our work focused on task effects on access to mental objects, we had to check whether task order modulated the observed interaction. In a final analysis, we considered task order effects in the three experiments with two different comparison tasks. In our experiments, the order of the two tasks was balanced across subjects. One possible effect of task order might have been that during the first part of the experiment, the subjects established a task set that, among other things, determined the level of maintenance. In this case, we would expect effects of the first task set on object switching in the second part: Object-switching costs in the semantic task should be larger if it was preceded by the locRec task, where semantics did not need to be maintained. If this was the case, the level of maintenance outside the focus would be open to strategic influences. This would contradict our assumption of losing semantic information outside the focus of attention.

A reanalysis of the data from Experiments 1, 3, and 4 did not confirm this alternative hypothesis: The RTs and object-switching costs in the semantic task were consistently lower when preceded by the locRec blocks, whereas there were no effects of task order on the locRec task. In

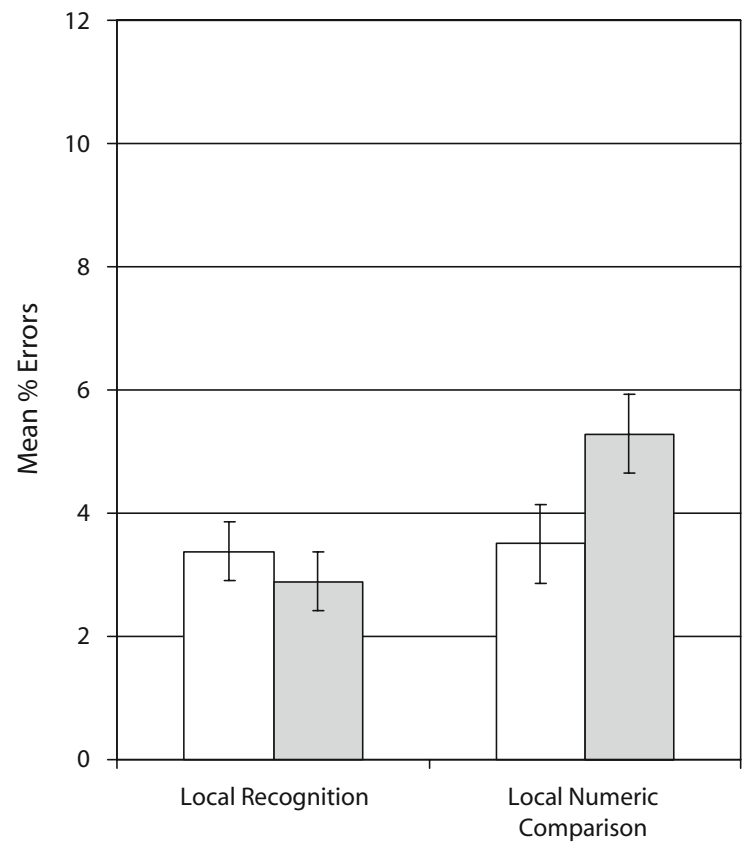

Task

Figure 6. Mean response times (RTs) and error rates for the two comparison tasks (local recognition and local numeric comparison) in Experiment 4 on same-object and object switch trials. 
an ANOVA including all three experiments, this pattern was indicated by a three-way interaction of object switch, task, and task order $\left[F(61,1)=11.00, p=.002, \eta^{2}=\right.$ $.15]$ that was not modulated by the between-subjects factor of experiment $(F<1)$. There appears to have been a general practice effect, rather than a carryover effect of the maintenance level: Performing the harder task after several object-switching blocks resulted in reduced objectswitching costs, as compared with performing it at the beginning of the experimental session. Garavan (1998) also reported reduced object-switching costs after training. Since we considered two different comparison tasks, practice must have affected a component that was common to both tasks; a candidate is object selection. The fact that practice effects were absent in the locRec tasks probably indicates a floor effect: Object-switching costs were probably already minimal, and no further savings of control resources through practice was possible. Practice effects on object switching in the semantic tasks support the assumption of limited resources that are shared between the (difficult) comparison task and object selection.

There was, therefore, an interaction of difficulty and object switching that affected object selection processes. However, object-switching costs in the semantic tasks were substantially higher, even after quite extensive object-switching practice (525 comparison trials, 300 object switches). Of course, we cannot exclude with absolute certainty the possibility that still more practice on object switching could eventually have eliminated this difference. But on the basis of the present evidence, we would argue that the interaction of task difficulty and object selection was not likely to have been the main cause of the differing object-switching costs between the two tasks. However, easier or practiced conditions might provide better estimates of the time required for feature retrieval processes, because they would be less overshadowed by general resource demands. The issue of estimating the relative contributions of object selection and feature retrieval to object switching should be pursued in further research.

\section{GENERAL DISCUSSION}

In our study, we investigated goal-directed access to mental objects in working memory. In the introduction, we pointed out that current accounts explaining such access by a single process of object selection providing the selected object for any upcoming mental operation (Oberauer, 2002, 2003) are probably not sufficient to adequately describe intention-guided manipulation of working memory information. Instead, getting mental objects from the direct-access region of working memory into the focus of attention for processing would be understood better as a dynamic combination of selection and retrieval processes determined by current task goals or processing intentions. According to this view, more specific assumptions concerning the properties of mental objects within and outside the focus of attention must be made. In contrast to Oberauer (2002), we assume (1) that a selected object is accessed specifically for the task to be executed on it (but not "any mental operation") and (2) that men- tal objects no longer receiving attention (as occurs after being removed from focus) lose information that cannot be maintained by quasi-automatic rehearsal processes. Therefore, accessing a new mental object for processing does not only require object selection: Retrieval processes become necessary, whenever the processing task relies on object features other than the features provided by maintenance rehearsal.

In support of this view, we reported four experiments in which the required object information (i.e., the kind of features required by the task) was manipulated, while conditions that could affect object selection (e.g., set size, memory materials) were kept constant. Object-switching costs were consistently larger when the comparison task required semantic object information, as compared with a locRec task.

\section{Maintenance in Working Memory}

At the beginning of this article, we made a quite strong assumption concerning the maintenance of mental objects currently not selected for processing: We suggested that these codes are available only as phonological codes. Several reasons led us to this assumption. First, selecting a mental object for the focus of attention means drawing attention away from the other candidate(s) to spend it on the selected object. As a consequence, since the whole relevant memory set has to be maintained successfully to perform an object-switching task, a process will be required that keeps all objects available in the total (or almost total) absence of attention. A process that supports the maintenance of verbal information in working memory and works almost automatically is subvocal rehearsal (Baddeley \& Hitch, 1974). However, subvocal rehearsal is assumed to continuously refresh the phonological representations of the elements of the memory set, or at least representations that are close to articulation plans (see Page et al., 2007). Mental objects can be accessed via these representations, which serve as retrieval cues for further object information.

In fact, the results of our experiments show that semantic information of a newly selected object appears not to be as rapidly available as its phonological identity, even though subjects know that, over the whole experimental block, semantic information will need to be accessed. This is consistent with the view that they are not able to maintain semantic information of mental objects outside the focus of attention and that a new object is initially available as a phonological code. If additional (e.g., semantic) features of the selected object are required by the task, they have to be retrieved or activated.

Although these strict assumptions are consistent with our results so far, they are an oversimplification - albeit an experimentally useful one-of the working memory mechanisms and should be put back into the general working memory context. In our experiments, we proposed phonological codes because subvocal rehearsal is a very typical and well-established maintenance strategy in verbal working memory. But even in our experiments, not all information was verbal. Bindings between shape or position cues and mental objects are not very likely 
to be rehearsed phonologically but must necessarily be maintained to accomplish the task (Oberauer, 2007). Depending on the context and the kind of bindings, we spend more or less attention on maintenance. As a consequence, the loss of semantic information outside the focus of attention is relative rather than absolute. Furthermore, the locRelC task probably evokes the use of visual strategies that make use of the mental number line (e.g., putting the mental object to the left or to the right of the 4), and the comparison might also be resolved via imagination. However, visual strategies do not seem to have a strong effect in our task, since they obviously cannot improve the availability of relational information outside the focus of attention. There are also no differences in object-switching costs between same- and different-relation comparison trials that can indicate a modifying influence of visuospatial codes. However, since there are very different kinds of working memory tasks and modalities, there must be different maintenance strategies that necessarily result in the maintenance of different kinds of object information. The general point we wish to make is that tasks differ in feature demand and that information provided through maintenance is not sufficient for the task at hand. Instead, it must be provided once a mental object is selected for the focus of attention. We could demonstrate this for subvocally rehearsed verbal material, but further studies are necessary to clarify whether these assumptions also hold for different working memory tasks. There are experimental results from an $n$-back task that point in a similar direction: Zhang (2006) found higher focus-switching costs in visual working memory with increasing complexity of the patterns to be processed. The increased costs might result from the need to retrieve more complex information from the selected object, because a more complex comparison is required. But the evidence is only indirect, since in this $n$-back task, memory demand, object access, memory updating, and comparison difficulty cannot be easily dissected.

Our assumptions are in line with models that assume that information about elements held in working memory are not fully available at retrieval, due to decay or interference that leads to the degradation of the respective code during retention. Therefore, retrieval of memory elements always includes a resource-demanding process of redintegration (Hulme, Newton, Cowan, Stuart, \& Brown, 1999; Schweickert, 1993) or reconstruction (Saint-Aubin \& Poirier, 2000) that "repairs" the degradation and provides the selected element for the required task. In these models, retrieval is based on the selection of a phonological trace that can be more or less degraded. We, in contrast, assume that the phonological code is refreshed by automatic rehearsal and is not very likely to be degraded very much (at least in our paradigm or, e.g., in mental calculation tasks with not more than three or four steps and intermediate results). What appears to degrade very quickly is semantic object information. So, when a new mental object for a semantic task is accessed, the retrieval of semantic features with the help of the phonological code takes timea process comparable to reconstruction or reintegration, despite the fact that, in this situation, the original phono- logical trace is assumed to be relatively intact, whereas the lost (nonphonological) object information has to be "reconstructed." This view is compatible with the reconstruction hypothesis, since it can be seen as its adaptation to an object-switching situation, whereas the model originally referred mainly to serial recall.

But there are approaches that assume semantic maintenance in verbal working memory, too. How do our assumptions and results relate to the idea of a semantic or conceptual short-term store (Haarmann \& Usher, 2001; see also Potter, 1993)? Haarmann and Usher (2001) showed, in a free recall task, that there is conceptual information available from short-term memory (namely, at recency-i.e., for the last part of the list, where retrieval from long-term memory is less likely to have an influence). Moreover, articulatory suppression did not diminish this semantic effect, suggesting a difference between phonological and semantic maintenance over the short term. Haarmann and colleagues proposed a semantic short-term memory that is individually limited in capacity and consists of a number of coactivated concepts and their relations that enable the online integration of information during higher level processing, such as speech comprehension (Haarmann, Davelaar, \& Usher, 2003; Haarmann \& Usher, 2001). Up to this point, we have associated phonological information with the maintenance of mental objects outside the focus of attention and semantic object information with processing within the focus. Haarmann and colleagues assumed semantic maintenance in short-term memory, but the capacity of this conceptual store was estimated at three to four concepts, which approximates the typical estimates of the maximum capacity of the focus of attention (see Cowan, 2001), and in the General Discussion section of their article, Haarmann et al. (2003) suggested that "semantic STM provides a system for maintaining concepts in or near the focus of awareness, enabling rapid access to stored concepts and rapid computation of relations among them" (p. 340). Phonological short-term memory serves, in their terms, as a back-up system if semantic online integration is not possible. This is in accordance with our view, insofar as it implies that semantic processing is closely related to the focus of attention and that the ability to provide semantic information depends on attentional resources. However, in contrast to our assumption (one mental object in the focus of attention), Haarmann et al. proposed a semantic focus of about four chunks.

The critical difference between our study and Haarmann et al.'s (2003; Haarmann \& Usher, 2001) probably lies in the kind of task applied. In our selective locRelC task, each step required the semantic processing of one object in order to produce a selective response. In this situation of single mental objects competing for selective access, there is no need for their online semantic integration. Rather, interference from nonselected objects has to be avoided, and it might be better to focus on one chunk while keeping the others in the backup system. In contrast, the conceptual span measure of Haarmann and colleagues is a pure memory task and relies on keeping as much information as possible available, without the need to select a single concept for processing. More recent results from experi- 
ments that required the access of more than one mental object (Oberauer \& Bialkova, 2009) suggest that focused attention forms chunks online. These chunks can be kept within the region of direct access as the broader focus of attention (Oberauer, 2002, 2007). This may be a possible mechanism for semantic integration in the semantic span task. However, although the semantic span results demonstrate the simultaneous availability of several semantic chunks, there still may be differences in accessing times during recall, when the chunks have to be selected serially. Such differences, in turn, could partly be a result of less available semantic information of chunks outside the focus of attention.

The fact that we found larger object-switching costs when the semantics of a new mental object had to be accessed does not necessarily imply that relational information is completely lost. There are results from another approach to investigating the availability of semantic information in verbal working memory that indicate the availability of not-attended semantic features for quite a long time after presentation and independent of intervening processing steps. Woltz and Was $(2006,2007)$ found priming effects of semantic categories that were explicitly not to be maintained in a memory task on a subsequent category comparison task. Furthermore, the available semantics appeared to be operation specific (i.e., if relevant and irrelevant memory exemplars were defined by the semantic category, there would be priming effects of categorical but not other semantic information). These studies do not call into question our conclusions concerning the reduced availability of semantic features of unattended mental objects in our task (where all objects were actively maintained). The unattended category was always less available than the category that was actively maintained, thus producing less priming. But the indirect priming effects found by Woltz and colleagues show that at least some semantic information is remembered. They suggest that information related to former processing steps remains in a state of availability that distinguishes it from unrelated memory information.

\section{Selection of Mental Objects in Working Memory Tasks}

Our last point refers to the concept of mental objects in working memory tasks. It was used first by Oberauer (2002), who described them as chunks held in an active state, independently of the task at hand. In our experiments, we showed that mental objects should be better considered with respect to the task for which they are accessed. So we use the term mental object in a literal sense for the units of memory information actually dealt with in a certain working memory task. Since mental tasks differ with respect to the specific information they require to be carried out, "the same" mental objects (e.g., the same numbers) can differ in their characteristics, depending on the operations they are subjected to. Consequently, there is also a qualitative difference between the mental object that is actually processed within the focus of attention and the objects currently not selected.
If we speak about task-specific object access, the question arises as to what the consequences of this task specificity are. In the introduction, we cited evidence that showed better availability of task-relevant information, as compared with task-irrelevant information, within one and the same object in the visual domain (Fanini et al., 2006). On the other hand, we cannot (and do not intend to) negate the importance of object-based attention in selection of objects for processing. In a recent work, $\mathrm{Bao}, \mathrm{Li}$, and Zhang (2007) demonstrated that for mental objects in working memory, switching between the same two features was completed more quickly when they were bound to one mental object, as compared with a condition in which they belonged to distinct mental objects. Moreover, switching between the features within an object took less time than did switching to the features of another object. These results strongly support an object-based view on access to mental objects: All the features of a selected object appear to be more available than the features of an object not currently selected. However, an intraobject feature switch also results in a time cost, suggesting that the formerly irrelevant feature is less available. On the basis of this evidence, one can assume that a mental object is selected as a whole but that the availability of specific features appears to be modulated by the current task. This is consistent with our idea of task-independent object selection plus taskspecific access to currently relevant object features. One possible mechanism would be that the selection of an object enables the activation of all the (sometimes partially degraded) features bound to it in the current task context. Our experiments demonstrated that not all object features are available after object selection and that the activation of semantic information takes time. Consequently, switching costs in the semantic task are higher. As a next step, it would be desirable to test whether the costs of an intraobject switch from the semantic to the more superficial feature or vice versa are asymmetrical. If all the features bound to the object are activated, superficial information should also be available after semantic features have been accessed. On the other hand, semantic features need not be available after superficial information is accessed, because they take longer to activate and the superficial processing task can be accomplished before they are provided. A further question that remains open is whether or under what circumstances selectivity among the features of one and the same mental object occurs.

\section{Conclusion}

Our experimental data at this point support the hypothesis of task-specific access to mental objects from verbal working memory, in contrast to a task-independent object selection process that provides selected mental objects for any upcoming operation. They are consistent with our assumption that in a typical working memory task, verbal mental objects outside the focus of attention are maintained as phonological codes that are refreshed continuously by subvocal rehearsal. Accessing a mental object for processing therefore includes selection among the phonological codes, as well as the retrieval of additional 
features of the selected object, if needed for execution of the task at hand.

To summarize, we understand our results as further evidence for a dynamic focus of attention in working memory. Access to mental objects cannot be considered a task-independent selection within a set of memory elements but is guided by the task goals of the individual. Consequently, the quality of one and the same mental object in the focus of attention varies with the demands of the current task.

\section{AUTHOR NOTE}

We thank Klaus Oberauer for many helpful comments on former versions of the manuscript. Thanks also to Martina Laschinski, Maria Wirth, Eva-Maria Ziethen, and Christine Umeh for collecting the data in Experiments 3 and 4 and Kate Könnecke for proofreading. Correspondence concerning this article should be addressed to S. Schwager, Institute of Psychology, Department of General Psychology, Humboldt University of Berlin, Rudower Chaussee 18, 12489 Berlin, Germany (e-mail: sabine .schwager@hu-berlin.de).

\section{REFERENCES}

BADDELEY, A. D. (1986). Working memory. Oxford: Oxford University Press.

BADDELEy, A. D. (2000). The episodic buffer: A new component of working memory? Trends in Cognitive Sciences, 4, 417-423. doi:10.1016/ S1364-6613(00)01538-2

BadDEley, A. D., \& Hitch, G. (1974). Working memory. In G. H. Bower (Ed.), The psychology of learning and motivation: Advances in research and theory (Vol. 8, pp. 47-89). New York: Academic Press.

BAO, M., LI, Z.-H., \& ZHANG, D.-R. (2007). Binding facilitates attention switching within working memory. Journal of Experimental Psychology: Learning, Memory, \& Cognition, 33, 959-969. doi:10.1037/0278 $-7393.33 .5 .959$

BERINGER, J. (1999). Experimental run time system. Frankfurt am Main: Berisoft Cooperation.

Carlson, R. A., Sullivan, M. A., \& Schneider, W. (1989). Practice and working memory effects in building procedural skill. Journal of Experimental Psychology: Learning, Memory, \& Cognition, 15, 517526. doi:10.1037/0278-7393.15.3.517

Cowan, N. (1988). Evolving conceptions of memory storage, selective attention, and their mutual constraints within the human information-processing system. Psychological Bulletin, 104, 163191. doi: 10.1037/0033-2909.104.2.163

Cowan, N. (1995). Attention and memory: An integrated framework. New York: Oxford University Press.

Cowan, N. (2001). The magical number 4 in short-term memory: A reconsideration of mental storage capacity. Behavioral \& Brain Sciences, 24, 87-185. doi:10.1017/S0140525X01003922

Engle, R. W., Tuholsky, S. W., Laughlin, J. E., \& Conway, R. A. (1999). Working memory, short-term memory, and general fluid intelligence: A latent-variable approach. Journal of Experimental Psychology: General, 128, 309-331. doi:10.1037/0096-3445.128.3.309

FANINI, A., NoBre, A. C., \& Chelazzi, L. (2006). Selecting and ignoring the component features of a visual object: A negative priming paradigm. Visual Cognition, 14, 584-618. doi:10.1080/13506280500195367

Garavan, H. (1998). Serial attention within working memory. Memory \& Cognition, 26, 263-276

Griffin, I. C., \& Nobre, A. C. (2003). Orienting attention to locations in internal representations. Journal of Cognitive Neuroscience, 15, 1176-1194. doi:10.1162/089892903322598139

HaArmann, H., Davelaar, E. J., \& Usher, M. (2003). Individual differences in semantic short-term memory capacity and reading comprehension. Journal of Memory \& Language, 48, 320-345. doi:10.1016/ S0749-596X(02)00506-5

HaARManN, H., \& Usher, M. (2001). Maintenance of semantic information in capacity-limited item short-term memory. Psychonomic Bulletin \& Review, 8, 568-578.
Hulme, C., Newton, P., Cowan, N., Stuart, G., \& Brown, G. (1999). Think before you speak: Pauses, memory search, and trace redintegration processes in verbal memory span. Journal of Experimental Psychology: Learning, Memory, \& Cognition, 25, 447-463. doi:10.1037/0278-7393.25.2.447

Jones, D. M., Macken, W. J., \& Nicholls, A. P. (2004). The phonological store of working memory: Is it phonological and is it a store? Journal of Experimental Psychology: Learning, Memory, \& Cognition, 30, 656-674. doi:10.1037/0278-7393.30.3.656

Kane, M. J., Hambrick, D. Z., Tuholski, S. W., Wilhelm, O., Payne, T. W., \& ENGLE, R. W. (2004). The generality of working memory capacity: A latent-variable approach to verbal and visuospatial memory span and reasoning. Journal of Experimental Psychology: General, 133, 189-217. doi:10.1037/0096-3445.133.2.189

Kessler, Y., \& Meiran, N. (2006). All updatable objects in working memory are updated whenever any of them are modified: Evidence from the memory updating paradigm. Journal of Experimental Psychology: Learning, Memory, \& Cognition, 32, 570-585. doi: $10.1037 / 0278-7393.32 .3 .570$

Kyllonen, P. C., \& Christal, R. E. (1990). Reasoning ability is (little more than) working memory capacity?! Intelligence, 14, 389-433. doi:10.1016/S0160-2896(05)80012-1

Lepsien, J., Griffin, I. C., Devlin, J. T., \& Nobre, A. C. (2005). Directing spatial attention in mental representations: Interactions between attentional orienting and working-memory load. NeuroImage, 26, 733-743. doi:10.1016/j.neuroimage.2005.02.026

Lepsien, J., \& Nobre, A. C. (2006). Cognitive control of attention in the human brain: Insights from orienting attention to mental representations. Brain Research, 1105, 20-31. doi:10.1016/j.brainres .2006 .03 .033

Li, Z. H., Sun, X. W., Wang, Z. X., Zhang, X. C., \& Zhang, D. R. (2004). Behavioral and functional MRI study of attention shift in human verbal working memory. Neurolmage, 21, 181-191. doi:10.1016/j .neuroimage.2003.08.043

Loftus, G. R., \& Masson, M. E. J. (1994). Using confidence intervals in within-subject designs. Psychonomic Bulletin \& Review, 1, 476490.

Naveh-Benjamin, M., \& Jonides, J. (1984). Maintenance rehearsal: A two-component analysis. Journal of Experimental Psychology: Learning, Memory, \& Cognition, 10,369-385. doi:10.1037/0278 $-7393.10 .3 .369$

Nobre, A. C., Coull, J. T., Maquet, P., Frith, C. D., VandenBerghe, R., \& Mesulam, M. M. (2004). Orienting attention to locations in perceptual versus mental representations. Journal of Cognitive Neuroscience, 16, 363-373. doi:10.1162/089892904322926700

Oberauer, K. (2001). Removing irrelevant information from working memory: A cognitive aging study with the modified Sternberg task. Journal of Experimental Psychology: Learning, Memory, \& Cognition, 27, 948-957. doi:10.1037/0278-7393.27.4.948

Oberauer, K. (2002). Access to information in working memory: Exploring the focus of attention. Journal of Experimental Psychology: Learning, Memory, \& Cognition, 28, 411-421. doi:10.1037/0278 $-7393.28 .3 .411$

Oberauer, K. (2003). Selective attention to elements in working memory. Experimental Psychology, 50, 257-269. doi:10.1026/1618 $-3169.50 .4 .257$

Oberauer, K. (2005). Control of the contents of working memory-A comparison of two paradigms and two age groups. Journal of Experimental Psychology: Learning, Memory, \& Cognition, 31, 714-728. doi: $10.1037 / 0278-7393.31 .4 .714$

Oberauer, K. (2007). Activation, binding, and selective access: An embedded three-component framework of working memory. In N. Osaka, R. Logie, \& M. D'Esposito (Eds.), The cognitive neuroscience of working memory (pp. 351-368). Oxford: Oxford University Press.

Oberauer, K., \& Bialkova, S. (2009). Accessing information in working memory: Can the focus of attention grasp two elements at the same time? Journal of Experimental Psychology: General, 138, 6487. doi: $10.1037 / \mathrm{a} 0014738$

Page, M. P. A., Madge, A., Cumming, N., \& Norris, D. G. (2007). Speech errors and the phonological similarity effect in short-term memory: Evidence suggesting a common locus. Journal of Memory \& Language, 56, 49-64. doi:10.1016/j.jml.2006.09.002 
PotTer, M. C. (1993). Very short-term conceptual memory. Memory \& Cognition, 21, 156-161.

SAINT-Aubin, J., \& PoIrIER, M. (2000). Immediate serial recall of words and nonwords: Tests of the retrieval-based hypothesis. Psychonomic Bulletin \& Review, 7, 332-340.

SCHWEICKERT, R. (1993). A multinominal processing tree model for degradation and redintegration in immediate recall. Memory \& Cognition, 21, 168-175.

Süß, H.-M., Oberauer, K., Wittmann, W. W., Wilhelm, O., \& ScHulze, R. (2002). Working-memory capacity explains reasoning ability — and a little bit more. Intelligence, 30, 261-288. doi:10.1016/ S0160-2896(01)00100-3

Voigt, S., \& Hagendorf, H. (2002). The role of task context for component processes in focus switching. Psychologische Beiträge, 44, 248-274.

Woltz, D. J., \& WAs, C. A. (2006). Availability of related long-term memory during and after attention focus in working memory. Memory \& Cognition, 34, 668-684.

Woltz, D. J., \& WAS, C. A. (2007). Available but unattended conceptual information in working memory: Temporarily active semantic content or persistent memory for prior operations. Journal of Experimental Psychology: Learning, Memory, \& Cognition, 33, 155-168. doi:10.1037/0278-7393.33.1.155

ZHANG, Y. (2006). Stimulus complexity, encoding, retrieval and focus switching. Unpublished doctoral dissertation, Syracuse University.

\section{NOTES}

1. RTs shorter than $300 \mathrm{msec}$ or exceeding the individual mean per condition by more than three standard deviations were excluded before the normalization.

2. The semantic comparison task used here requires the determination of the relations of the selected mental object and of the displayed digit to the number 4 before execution. One might argue that the increase in object-switching costs in this task is not solely due to the retrieval of semantic features but results from an additional mental operation on object switch trials: On same-object trials, the relation of the mental object to the number 4 has been just determined, whereas on object switch trials, determination of the relation is not primed this way. Although we cannot rule out such a contribution, we do not consider the decision process a main source of object-switching costs. Since one-digit numbers are highly familiar and, in addition, the six digits used occur many times over the experimental condition, their relations to the number 4 are likely to be retrieved from memory, rather than determined by a demanding operation on the mental number line. Indirect support for this claim is provided by Experiment 2: Object-switching costs are not lower when no relations are required to be newly determined. Consequently, we assume that the category of semantic features may include the relational information, instead of being restricted to the digits' numerical values.

3 . There might be a similar access priming effect of relational information, but this was not the case (object switch $\times$ response interaction, $F<1$ ).

4. We found reduced object-switching costs on different trials when nonset digits were presented as stimuli [object switch $\times$ set interaction, $\left.F(22,1)=46.07, p<.001, \eta^{2}=.68\right]$ and, after exclusion of nonset stimuli, reduced object-switching costs on same trials [object switch $\times$ response interaction, $F(22,1)=48.24, p<.001, \eta^{2}=.69$ ]

5. In the locRec task, same trials were excluded from analysis. In the locNumC task, stimulus digits smaller than the smallest or larger than the largest member of the memory set could, in principle, be responded to directly without accessing the specified object. No evidence for this alternative strategy was found when contrasting object-switching effects for the edging digits ( 1 and 9 ) with those for the remaining stimuli (object switch $\times$ stimulus class interaction, $F<1$ ). All trials were subjected to statistical analyses.

(Manuscript received June 10, 2008; revision accepted for publication June 30, 2009.) 